\title{
¿INGRESAN LOS ALUMNOS EN LA UNIVERSIDAD CON UN ADECUADO DESARROLLO DE LOS NIVELES DE RAZONAMIENTO?
}

\author{
BRAGA, I.L. \\ Departamento de Física. Universidad de Concepción. Concepción (Chìe)
}

\begin{abstract}
SUMMARY
Most of the students that enrol in the area of physical sciences and mathematics, in the Universities in Chile fail in the first courses of physics and mathematics. To try to solve this problem a course on intellectual development was devised; the contents and outcome of this course are presented.
\end{abstract}

\section{INTRODUCCION}

El hecho de que más del $45 \%$ de los alumnos que ingrcsan a la Universidad, en el área de las Ciencias F́. sicas y Matemáticas fracasen al cabo del primer año, ya sea en Física, Matemáticas o ambas asignaturas a la vez, no hace más que acumular evidencias de que una gran mayoria de los alumnos que ingresan a los primeros años de esta área (correspondiente a las carreras de Ingenieria y licenciaturas), no presentan un adecuado nivel de razonamiento (Renner y Lawson 1973), (Workschop on Physics 1975), (Renner 1976), (Braga 1980), (Robbins 1981), (Arons 1981), que les permita ingresar a los cursos introductorios de Matemáticas como de Física, con los prerrequisitos mínimos de habilidades intelectuales correspondientes a los patrones de razonamiento.

El primer objetivo de este artículo, consiste en mostrar algunos resultados provenientes del trabajo de investigación denominado «Identificación de las causas más relevantes que inciden en los bajos rendimientos de los primeros cursos de Fisica y Matemáticas en el sistema universitario chileno", (desarrollado en el De. partamento de Física de la Universidad de Concepcion) y que avala la afirmación inicial acerca de los niveles de razonamiento presentado por los alumnos que ingresan a dichos cursos introductorios.

El segundo objetivo es dar a conocer un modelo de curso destinado exclusivamente a producir rápidos cambios en los niveles de razonamiento de los jóvenes universitarios y que ha mostrado ser bastante efectivo al aplicarlo en forma experimental en la Universidad de Concepción.

\section{EVALUANDO LOS NIVELES DE RAZONA- MIENTO DE ENTRADA}

Para evaluar el nivel de razonamiento de entrada de los alumnos, diseñamos y validamos un instrumento, basándonos en las pruebas originales del propio Piaget (Piaget e Inhelder, 1972) y en algunas otras que han sido utilizadas en otros países con tal fin, (Lawson, 1978), (Longeot, 1965), (Raven, 1973).

La prueba mide el grado de dominio del aiumno en los siguientes tipos de razonamiento: Razonamiento de conservación, falta de información en una linea de razonamiento, cadenas de razonamientos, razonamiento hipotético deductivo, razonamiento tipo control de variable, etc. Esta consta de dos partes, la primera es simplemente de trabajo con papel y lápiz y la segunda parte debe ser administrada personalmente a cada alumno en el laboratorio (en el apéndice A, se consigna una de las pruebas utilizadas para hacer la medición del nivel de razonamiento).

En la tabla 1, se consigna la información relativa al nivel de razonamiento de los alumnos que ingresaron a la Escuela de Ingenieria de la Universidad de Concepción, durante los anos que se indican.

Como criterio para discriminar entre dominio y no dominio, se consideró que un alumno domina un determinado tipo de razonamiento si éste presenta como mínimo un $55 \%$ de eficiencia en el desarrollo de la o las situación (es) propuestas para medir tal tipo de razonamiento en especial.

Al analizar los resultados presentados en la tabla 1, se observa que la mayoria de los alumnos carecen de un

ENSEÑANZA DE LAS CIENCIAS, 1987, 5 (1), 16-26 
Tabla 1

NIVEL DE RAZONAMIENTO DE ENTRADA

\begin{tabular}{|c|c|c|c|c|c|c|c|c|}
\hline TIPO OE RAZONAMIENTO & $\begin{array}{l}1978 \\
(225)^{\star}\end{array}$ & & $\begin{array}{l}1979 \\
(243)^{*}\end{array}$ & & $\begin{array}{l}1480 \\
(328)\end{array}$ & & $\begin{array}{l}1982 \\
(576)^{*}\end{array}$ & \\
\hline & $\begin{array}{c}\text { NO } \\
\text { DOMINA }\end{array}$ & DOMINA & $\begin{array}{c}\text { MO } \\
\text { DOMINA }\end{array}$ & DOMINA & $\begin{array}{c}\text { NO } \\
\text { DOMINA }\end{array}$ & DOMINA & $\begin{array}{c}\text { WOO } \\
\text { CONINA }\end{array}$ & DOMINA \\
\hline & $\frac{1}{6}$ & $\%$ & $\%$ & $\frac{1}{6}$ & 8 & 8 & T & 6 \\
\hline CONTROL DE VARIABLE & 91 & 9 & 88 & $\sqrt{2}$ & 92 & $T$ & 90 & 10 \\
\hline RAZONAMIENTO CONSERVACIOK & 68 & 32 & 85 & 15 & 81 & T9 & 75 & 25 \\
\hline $\begin{array}{l}\text { FALTA INFORHACION EN UKA } \\
\text { IINEA OE RAZONAMIENTO }\end{array}$ & 78 & 22 & 74 & 26 & 80 & 20 & 68 & 32 \\
\hline $\begin{array}{l}\text { CADENA DE RAZONRAIIENTOO } \\
\text { ( } 3 \text { PASOS) }\end{array}$ & 92 & 8 & $9 !$ & 9 & 85 & 15 & 82 & 18 \\
\hline $\begin{array}{l}\text { QRAZONAMIENTO RIPOTETICO } \\
\text { DEDUCTIVO }\end{array}$ & 79 & 21 & 83 & 17 & 85 & 15 & 79 & 21 \\
\hline $\begin{array}{l}\text { PAZOKARIENTO OE PROPORCTO- } \\
\text { NALIOAD }\end{array}$ & 75 & 25 & 82 & 18 & 81 & 19 & 67 & 23 \\
\hline $\begin{array}{l}\text { RAZONAWTERTO INDUETTVO EN } \\
\text { DESARROLLO DE MODELOS }\end{array}$ & 80 & 20 & 85 & 15 & 88 & \pm 2 & NO EV & VALUADO \\
\hline $\begin{array}{l}\text { RAZONANIENTO OEDUCTINO EN } \\
\text { LA PREDICCION DE CONSE- } \\
\text { CUENCIAS }\end{array}$ & 87 & 13 & 90 & 10 & 91 & 9 & 84 & 16 \\
\hline
\end{tabular}

* Los $\mathrm{N}$ entre paréntesis corresponden al $\mathrm{N}^{*}$ de alumog ingresados a $1 \mathrm{a}$ carrera

de Ingenierfa en la universidad de concepcion en los años indtcados.

desarrollo adecuado de los niveles minimos de razonamiento, para desenvolverse en un curso de Física universitario, con algunas posibilidades de éxito. Conclusiones similares se encuentran en estudios realizados en EE.UU. y en Europa (Winnon, 1971); (Robbin, 1981). A la luz de estos resultados es perfectamente explicable entonces, los pésimos rendimientos de los primeros cursos de Fisica en las Universidades.

Para encarar la problemática que presentan los alumnos que ingresan a la Universidad, diseñamos un curso destinado especialmente a promover el desarrollo de los niveles de razonamiento, y cuya sinopsis, forma de trabajo, resultado de su aplicación, implicaciones y conclusiones daremos en la siguiente parte de este articulo.

\section{CURSO PARA DESARROLLAR NIVELES DE RAZONAMIENTO DE LOS ALUMNOS QUE INGRESAN A LA UNIVERSIDAD}

En el disento de este singular curso, se ha tenido en cuenta especialmente el trabajo desarrollado por el psicólogo Jean Piaget, quien ha estudiado ampliamente to relativo al desarrollo del razonamiento en los jóvenes.

ENSEÑANZA DE LAS CIENCIAS, 1987, 5 (1)
Se desprende del trabajo de Piaget que el desarrollo de los niveles de razonamiento es sólo posible a través del proceso que él llama de auto-regulación: siendo cruciales en este proceso los factores de experiencias Fisica y transmisión social.

Teniendo en cuenta lo anterior, se diseñó el curso, de modo que se demande a los estudiantes una contínua y activa participación en cada una de las actividades puesto que, por lo general, los cambios de los patrones de razonamiento son algo que no se puede aprender leyendo un libro sino más bien mientras el estudiante está realizando y analizando los resultados de su propia experiencia, es decir, mientras trabaja activa y directamente con los fenómenos (curso enteramente activo).

El curso fue también diseñado de modo que no le demande en absoluto al alumno aprender una serie de contenidos sino que, más bien se considera a estos como un simple medio para organizar la serie de actividades, con el fin de conseguir el desarrollo de los diferentes niveles de razonamiento.

Para darle una cierta organicidad al curso, se eligió usar como columna vertebral de él, la estrategia correspondiente a la serie de los procesos científicos, cuya utilización contribuye enormemente al objetivo del curso. 
En el apéndice $B$ se consigna la sinopsis de uno de los cursos desarrollados con este fin. Alli encontrará en la primera columna, los tipos de razonamiento que se pretende desarrollar conjuntamente con el proceso que le sirve de base y, en la segunda columna, encontrará la correspondiente situación problemática que el alumno debe resolver.

\section{DESCRIPCION DEL CURSO}

El curso se desarrollará en base a tres tipos de actividades relacionadas mutuamente entre ellas, estas son sesiones de laboratorio, sesiones de coloquio y sesiones generales.

\subsection{Las sesiones de laboratorio}

Tienen como finalidad primordial proporcionar al alumno la oportunidad de:

- Que pueda manipular en el laboratorio los elementos necesarios, de modo tal que consiga familiaridad con los entes concretos, para a posteriori llegar a desarrollar abstracciones a partir de la información concreta por él procesada.

- Que se desenvuelva en forma grupal, de modo que en conjunto puedan planificar, realizar y analizar una secuencia de pasos destinados a resolver las situaciones problemáticas planteadas.

- Que desarrolle la capacidad de sintesis a través de la elaboración de la comunicación del trabajo realizado por él.

Estas sesiones han sido planificadas de modo que un docente trabaje con un grupo de 24 alumnos como máximo, divididos en grupos de 4 alumnos cada uno, trabajando durante 3 horas semanales. Ellas se desarrollarán principalmente en base a situaciones problemáitcas a resolver. (En el apéndice $C$ se presenta una actividad del tipo desarrollada en las actividades de laboratorio).

\subsection{Las sesiones de coloquio}

Son también de trabajo netamente grupal, en la cual cada profesor maneja el mismo grupo que para las sesiones de laboratorio y donde se realizan fundamentalmente las siguientes actividades:

- Revisión de la comunicación del trabajo realizado en el laboratorio, por medio de los mismos alumnos.

- Análisis critico de los resultados de los experimentos.

- Desarrollo de una profusa ejercitación de los diferentes tipos de razonamiento, mediante situaciones problemáticas relacionadas con las actividades realizadas en las sesiones de laboratorio. (En el apéndice D se presenta un tipo de material utilizado en las sesiones de coloquio).

\subsection{Las sesiones generales}

Son la parte menos importante del curso y se han incorporado más que nada como un medio para darle una visión de conjunto a este. En ellas se desarrollan entre otras, las siguientes actividades: motivación al trabajo a realizar durante la semana, análisis crítico de las conclusiones de algunas experiencias, planteamiento de problemas a analizar para contribuir al desarrollo de los niveles de razonamiento.

Semanalmente los alumnos, primero, deben asistir a las sesiones generales, luego, asistir a la sesión de laboratorio y, por último, asistir a la sesión de coloquio respectivamente.

\subsection{Evaluación}

La evaluación del progreso en los diferentes niveles de razonamiento, se va realizando periódicamente durante la realización del curso, mediante una serie de pruebas disenadas especialmente para tales fines. Cada una de estas pruebas de razonamiento (de carácter formativo), le informa al alumno acerca del estado de desarrollo en cada uno de los tipos de razonamiento considerados.

Las pruebas a que son sometidos los alumnos durante el desarrollo del curso van aumentando paulatinamente su grado de dificultad, ya que ellas van midiendo cada vez niveles más superiores de razonamiento.

La prueba final es una medida del nivel de razonamiento alcanzado por cada alumno de este curso.

Algunas de las pruebas a que son sometidos los alumnos durante el curso, deben realizarse solamente con papel y lápiz, otras en canbio, como por ejemplo la de control de variables, corresponde a una situación totalmente experimental; ya que solamente a través de su realización se revela el nivel de razonamiento alcanzado.

\section{RESULTADOS}

En los gráficos de la fig. I se consignan los resultados comparativos de los niveles de razonamiento de entrada y salida, de los alumnos que prosiguieron los cursos destinados especialmente a desarrollar la capacidad de razonamiento, realizado durante los aftos 1978 y 1979, en que se probó si el curso logra o no producir cambios significativos de los niveles de razonamiento, de los alumnos que ingresan a la Universidad de Concepción, a las carreras de Ingenieria de esos años.

Como puede apreciarse de la información dada, se observa un significativo desarrollo de los niveles de razonamiento.

ENSEÑANZA DE LAS CIENCIAS, 1987, 5 (1) 
figura 1

Gráficos comparativos de los niveles de zazonamiento de entrada y salida

ANO 1978

CONTROL DE VARIAB:E

RAZONAMIENTO CONSERVACION FALTA INFORMACION EN LI NEA DE RAZONAMIENTO

CADENA DE RAZONAMIENTO

PAZONAMTENTO MIPOTETICO DEDUCTIVO

RAZONAMIENTO DE PROPOR CIONALIDAD

RAZCNAMIENTO INDUCTIVO EN DESARROLLO DR MOOELOS

RAZONAMTENTO DEDUCTIVO PKEDICCION OE CONSECUEN CIAS DE UN MODELO

ANO 1979

CONTROL DE VARIABLE

RAZONAMIENTO CONSERVACION FALTA INFORMACION EN LI NEA DE RAZONAMIENTO

CADENA DE RAZONAMIENTO

RAZONAMIENTO HIPOTETICO DEDUCTIVO

RAZCNAMIENTO DE PROPOR CIONALIDAD

RAZCNAMIENTO INDUCTIVO EN DESARROLLO DE MODELOS

RAZCNAMENTO DEDUCTIVO PREDICCION DS CONSECUEN CIAS DR UN MODELO
ENTRADA

NIVEL EE DOMINIO

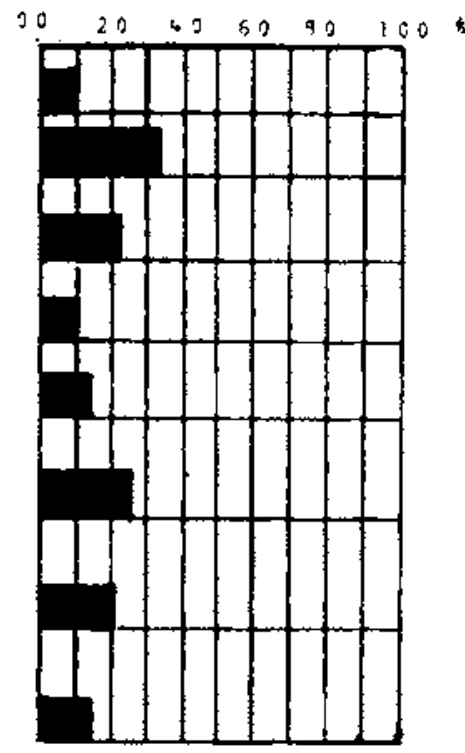

ENTRADA

NIVEL DE DOMINIO

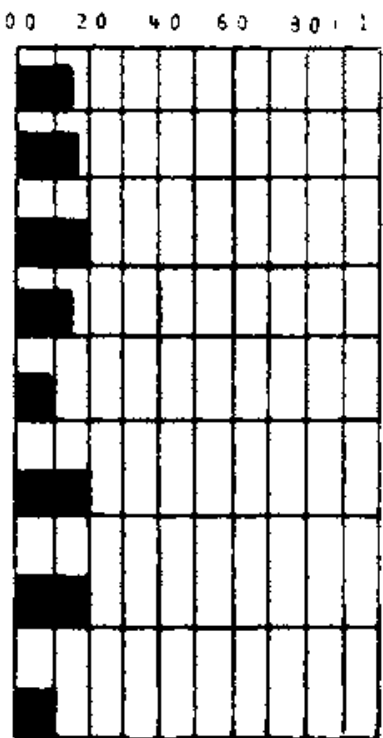

SAIIDA

NIVEL DE DOMINIO

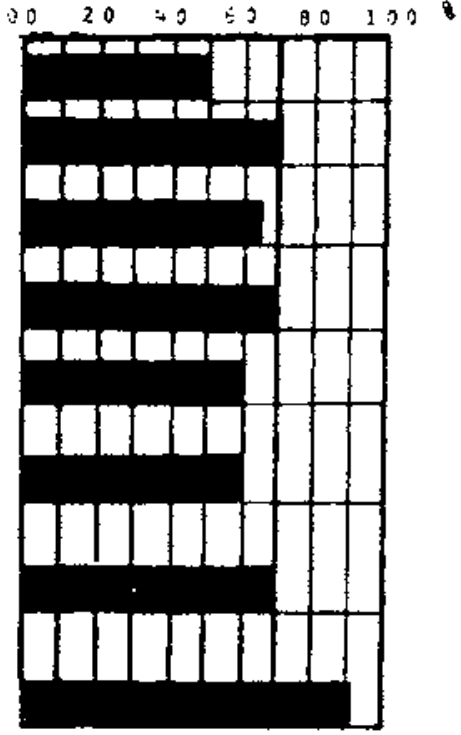

SAIIDA

NIVEL OE DOMENIO

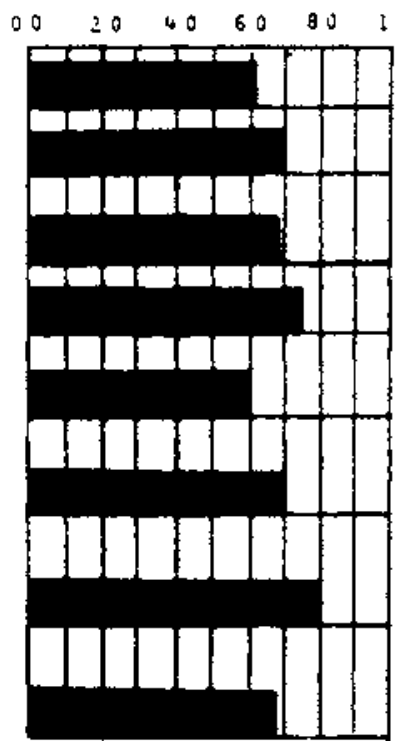


Con el objetivo de poder analizar el desenvolvimiento académico de los alumnos que cursaron estos cursos y poder determinar en definitiva si las estructuras de razonamiento desarrolladas por los alumnos, están disponibles para ser utilizadas por éstos en los siguientes cursos, disenamos un exhaustivo programa de seguj. miento, con el fin de poder detectar las dificultades con que se encontraron los alumnos, a fin de hacer los cambios necesarios y ajustes correspondientes a los próximos cursos de este tipo. Nuestro análisis se centró especialmente en los cursos de Mecánica que estos alumnos deben seguir a continuación de este curso. En los gráficos de la fig. 2 se compara el rendimiento final de los cursos de Mecánica de 1975 a 1977, cuyos alumnos ingresaron directamente al curso de Mecánica con los rendimientos de los alumnos de Ingeniería, que previamente cursaron el curso de desarrollo de las capacidades de razonamiento (de 1978 adelante).

Es importante dejar expresa constancia que el programa del curso de Mecánica no sufrió variación alguna por la implementación del curso de desarrollo de las capacidades de razonamiento, todavia más, los profesores que lo dirigian no cambiaron mientras se hizo la experiencia de implementación de estos cursos especiales.

\section{CONCLUSIONES GENERALES}

Al evaluar el inmenso cúmulo de experiencias e información recogida desde la génesis de este curso hasta su puesta en marcha y evaluación final, podemos mencionar las siguientes conclusiones de importancia:

1) Es posibie diseñar cursos para producir rápidos desarrollos en los niveles de razonamiento de una gran mayoria de los alumnos que ingresan a la Universidad, al área de Matemática y Física. Nuestra experiencia muestra que con un curso de este tipo (de un semestre de duración) sólo es posible lograr un significativo de. sarrollo de las estructuras de razonamiento en un por- centaje que bordea el $73 \%$ de los alumnos que ingresan a la Universidad.

2) Los cursos que se diseñan deben tener en cuenta los factores fundamentales que prescribe la teoria de Pia. get, para el desarrollo del razonamiento.

3) Se producen notables meioras en los niveles de rendimiento de los cursos convencionales de Física, si previamente se somete a los alumnos a cursos intensivos, destinados a completar su nivel de razonamiento.

4) Cómo el alumno tiene que realizar un número de actividades experimentales que él debe de informar. Se producen mejoras notables en el nivel de comunicación de los alumnos.

\section{DIFICULTADES ENCONTRADAS AL REA- LIZAR ESTOS CURSOS}

\section{A) En el ambiente docente:}

- Reticencia para hacer un curso sin contenido especifico.

- Dificultad para convencer a autoridades de por qué hacer un curso para desarrollar las capacidades de razonamiento.

- Dificultad para hacer cambiar la mentalidad de los docentes y permitir que los alumnos piensen y puedan desarrollar su nivel de razonamiento.

- Dificultad para formar equipos docentes dispuestos a trabajar con una modalidad diferente de un curso tradicional.

\section{B) En el ambiente estudiantil:}

- Dificultad para convencer a los alumnos que los contenidos mismos no son importantes sino sólo un medio para producir los desarrollos deseados.

- Dificultad para hacer pensar a los alumnos, ya que ellos quieren más que nada memorizar y luego repetir una información.

figura 2

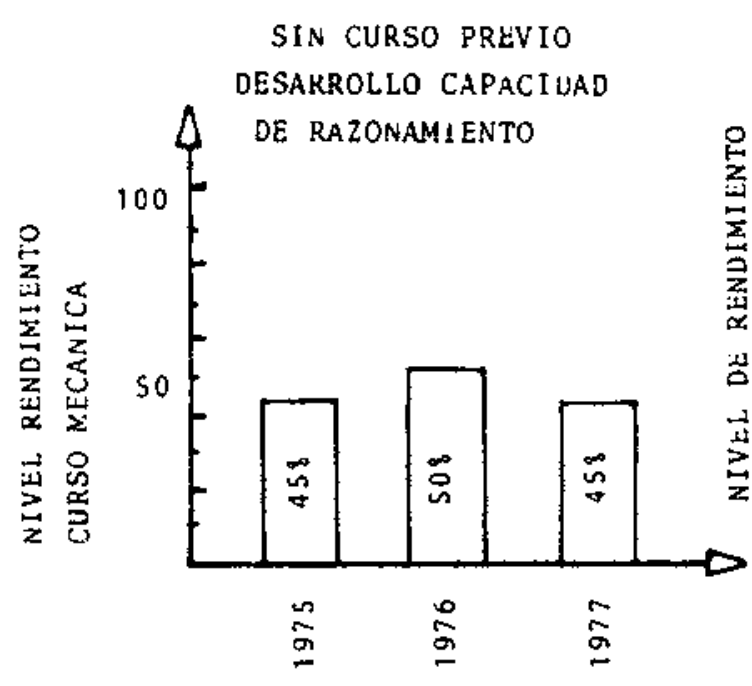

20

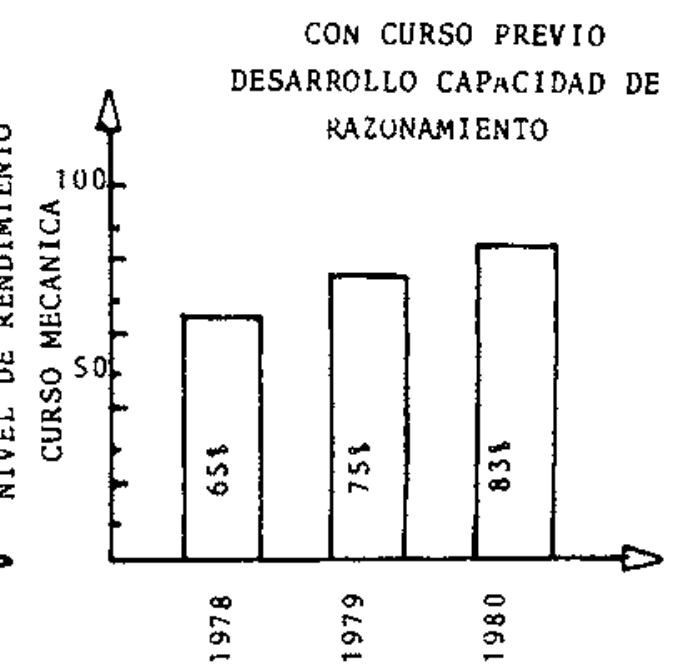

ENSEÑANZA DE LAS CIENCIAS, 1987, 5 (i) 


\section{DIRECTRICES FUTURAS}

Como los resultados obtenidos han sido tan promisorios, hemos realizado un detenido estudio de cada una de las dificultades detectadas para poder minimizarlas o eliminarlas, con el objeto de que iniciativas de este tipo puedan desarrollarse en distintas Universidades chilenas o latinoamericanas, para así llegar a mejoiar sustantivamente los rendimientos de los primeros cursos universitarios de Física y Matemáticas.

Sin embargo, como se menciona en la enumeración de dificultades, un "curso como el descrito", que es un curso que exclusivamente desarrolla el nivel de razonamiento, cuesta mucho introducirlo en cualquier $\mathrm{cu}$ - riculum de las careras de Ingenieria y Ciencias, ya que estas áreas tienen de por sí, un plan recargado de cursos y además, están limitados por el tiempo.

Teniendo muy presente esta importante limitación, nos encontramos avocados al diseño de cursos de Física, en los cuales se desarrolla dentro del contexto mismo del curso de Física, los niveles de razonamiento superiores.

Este enfoque conjunto presenta un desafío mayor, ya que es preciso resolver una difjcil problemática para congeniar el desarrollo lógico de la disciplina misma, con el desarrollo de los niveles de razonamiento que se desea alcanzar.

\section{REFERENCLAS BIBLIOGRAFICAS}

ARONS A., 1981 Thinking, Reasoning and Understanding in Introductory Physics course The Physics Teacher, P.P. $167-172$.

BRAGA L., 1980. Evaluando las Habilidades Intelectuales de los alumnos que ingresan a la Carreras de Ingenieria en las Universidades chilenas. Publicación interna. Facul. tad de Ciencias. Universidad de Concepción.

LAWSON A.E., 1978. The development and Validation of classroom test of formal reasoning. Journal of Research in Science Teaching 15. pp. 11-24.

LONGEOT F., 1965. Test to atte mpt the development logi$\mathrm{cal}$ thinking on Piagetan line, Bulletin del Institute $\mathrm{Na}$ tional D'Etdue. pp. 219-235.

PIAGET e INHELDER, 1972. De la lógica del nitho a la lógica del adolescente (Paidos) (Buenos Aires).

RAVEN, 1973. The development of a test of Piaget's logical

ENSEÑANZA DE LAS CIENCIAS, 1987, $s$ (1) operations, Science Education, 57, pp. 377-385.

RENNER J., LAWSON A., 1973. Promoting Intellectual Development Through Science Teachin The Physics Teacher pp. 273-276.

RENNER J., 1976. Significant Physics content and intellectual development cognitive development as a result of interaching with Physics content. American Journal of Physics 44 pp. 218-225.

ROBINN R., 1981. Improving student reasoning skills in Science classes. Engineering Education pp. 208-212.

WINNON J., 1971. Are colleges concerned with intellectual development American Journal of Physics. pp. 1047-1059.

WORKSCHOP on PHYSICS, 1975. Teaching and Development of Reasoning, edited F.P. coller (AAPI, Story Brook N. Y.), 


\section{APENDICE A}

Prueba: Medictón del desarrollo intelectual (Parte A)

AP. PATERHO

A. Matehno

NOKBRE

DURACION 120 HINUTOS

TN5TACCTDNES: Esta Prusba tiene por objaco analizar el estado de demarrollo Lotel cetual do entrafa, en termino da su capacidad de razona elonec que US. dabers abordar.

para resolver cada tituacion. uster dispone del espa-

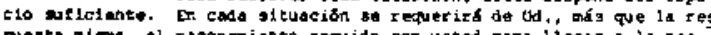

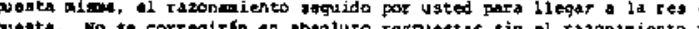
tundesentescion.

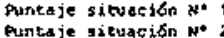

punta jo situseion $\mathrm{N}^{*}$

partaje gituseion $\mathrm{N}^{*}$

Puntaje aituacion $\mathrm{N}^{*}$

Pintajo gituacion n. $^{*}$

Purtaje gibuacion $\mathrm{H}^{\circ}$.

Rintaje sibuseion $n$

Puntaje situacion $\mathrm{N}^{*}$ in

SIruncion 1

Suponga que termos dos cuerpos marizos, de difezente forma luno exlindrico y al atro elffirleol. pero. de iguales volvisones y de o

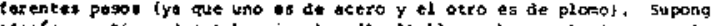

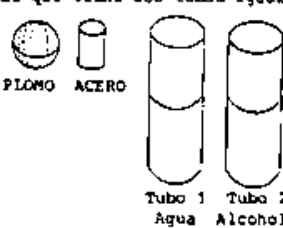
uno con agua y al ctro con alco

Ahors, si se inerodvee el cillin dro de acero en el tubo 1 y la iesmo sersin los niveles de los líguidos en los kubos?. Exply que su respuest. ind 1 edndo $\mathrm{el}$

Aquad Aleonol

Situacion 2: suponga gue gn thene una datro de sluminio de $25 \mathrm{con}$ de longitud, la cusi pesa in y una barra de cabre del aiseso diambero y langitud, is cual pesa $7.5 \mathrm{~N}$.

S1 ge cores un pedato de la barra de alusdalo ase pe sa $1 \mathrm{~N}$ y un podaso eactantente de igual longitud de la barta de cobre. ceudnto pesaź of trozo de cobra que queda datputs de hacter ol corto? Explique, coso $11 \mathrm{eq} 6$ a su respuesta. situaciow 3: un volumen de $30 \mathrm{cos}^{3}$ de agua ge ariade $350 \mathrm{~cm}^{3}$ de atu Trats de potar fortular una poible explicactón te ofL. rorprentente insisto

.1 correceat zesan podría parar a pruebe we explicación pars ver ei

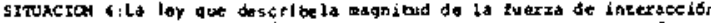

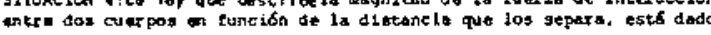
por

$$
F+\frac{2_{1} z_{2}}{R^{2}}
$$

Oonete $\mathrm{A}=\mathrm{ete}$

$z_{1}$ y $z_{2}$ propiodsoes do los cuerpor

Suptigase stars que cuando low cuexpas con propiedodes

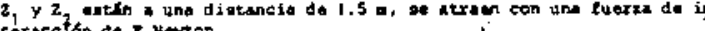

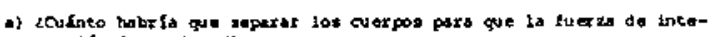
cacelsn uara jo ris

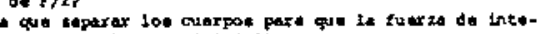

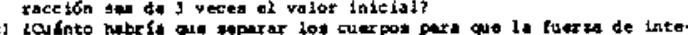
raceion fuara caror

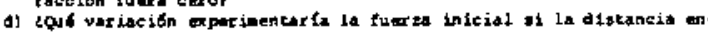

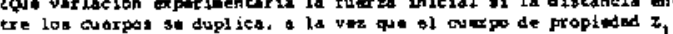

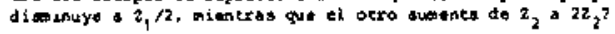

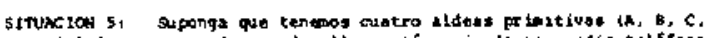

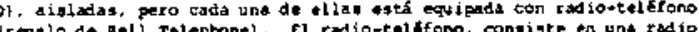
tran ivora y en una radio receptors. rodas lat sldess se encrentran

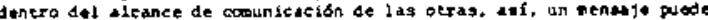
acr onvisto dir oct apente tentre dol abtacionts cuslesquiera, cuyos ta

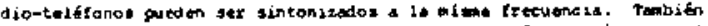

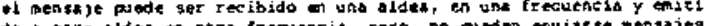

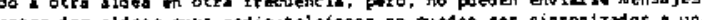

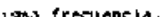

\begin{tabular}{|c|c|}
\hline$(1)$ & 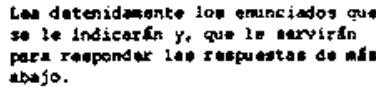 \\
\hline (1) & 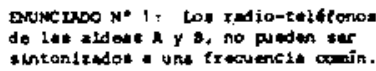 \\
\hline
\end{tabular}

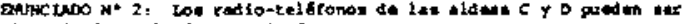

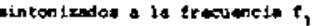

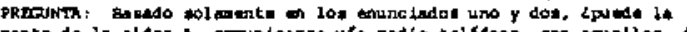

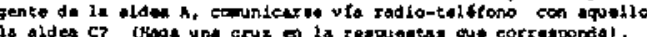

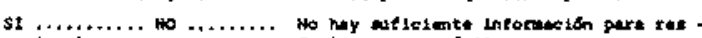

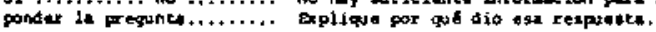

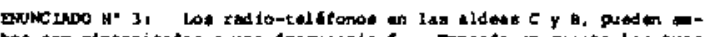

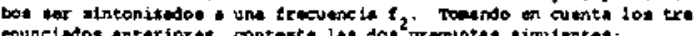

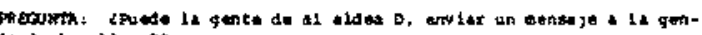
Ao de la aldes 8 ?

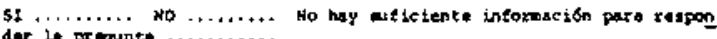
Expl lque por ols ofo ex..... regpuecte

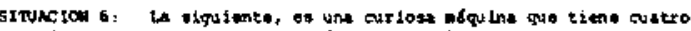

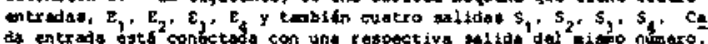

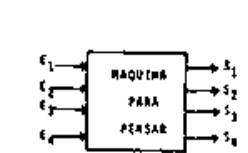

Constdere que las aldees astin rusigutant: tormat

- Las latros introducidas on $\mathrm{C}(1)$. salen oor la galfis s(1) isverti das to derocha a brovierdo. Las latxal incroducious on it? salen por ta galida $5(2)$ al re

- Las latras inkroducidas en E(3) soler por la salida S(3) al cevfo

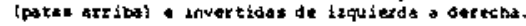

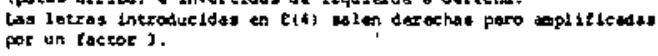

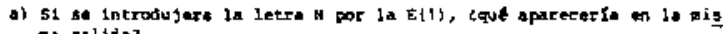

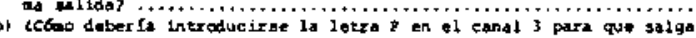
por lo or ma ealida cono so vo nomalmente?

c) ectemo ge observaris la letra $z$ an la saide oi ge la introduct por

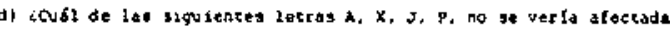

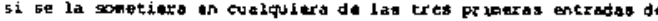
La caja?

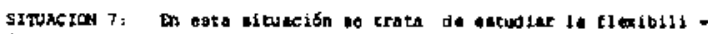
dad do un conjunto de varillas. eppecialmepte on lo gue se relaciona a Pora sesponder las alguitentes ptegunkas debe do conniserar el siguitite conjunto de varilisas.

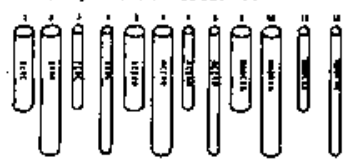

A cruslet pares de varitlas puede Ud, comparax pera encontrar af las

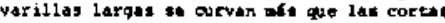

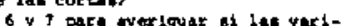

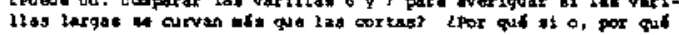

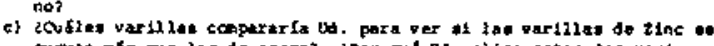

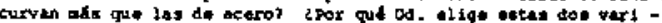

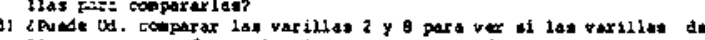

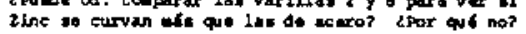

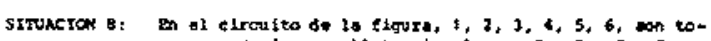

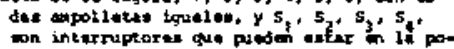

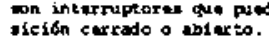

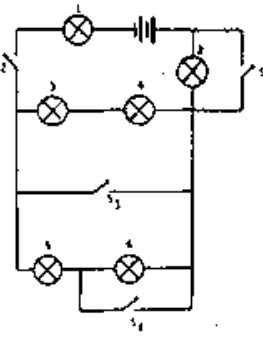

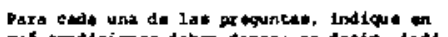

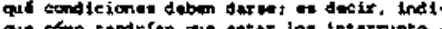

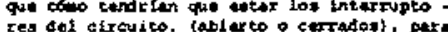

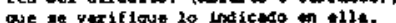
LQub condiclones deben darko para que! a) solo mejerds una mpolletar $s_{1} \ldots \ldots, s_{2}, \ldots \ldots, s_{3} \ldots \ldots, s_{4} \ldots \ldots \ldots$ b) solo dor apolleth meterden: $s_{1} \ldots \ldots \ldots s_{2} \ldots \ldots, s_{3} \ldots \ldots, s_{4} \ldots \ldots$.

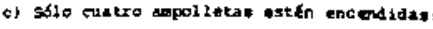
$s_{1} \ldots \ldots \ldots s_{2} \ldots \ldots \ldots s_{1} \ldots \ldots \ldots s_{8} \ldots \ldots \ldots$ 
d) sunquna encendida:

$s_{1} \ldots \ldots, s_{2} \ldots \ldots, s_{3} \ldots \ldots, s_{4}, \ldots \ldots$.

e) S610 lo ampollete 1 y 6 encitandan

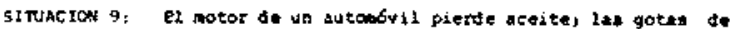
acerte caen al carino a razón de uhs por cass 2 segundoa. En ol diograsa de má abajo, ge muestran lap gotas de aceita dejadas on el camino thaturalmente no an ol tramaño noturall. La manchas qrandas repretentan gran cantidad do tceito. La linea de krazos o c, signtics que hay una gran distancia entros y $\mathrm{C}$ on ia Jido contabilizadar y similemente py to DE Y FG. $\quad \cdots .+$; -

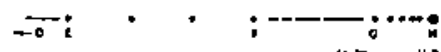

a) LQje inferencis podrís hacer acarca del moviaiento del auto donde hay grandes manthas do sceite?

b) isería posible determinar el timpo que demoro el auto en $1 x$ de $C$

lqué inferenciss potris nacor teapecto al movimiento del auto en el trayecto $A B$ G GH?

Si las goess de aceite en la posición CD, eetón sogaradas por 1 me

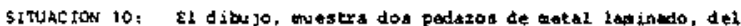
mi mo espesor y de la mi ma brea.

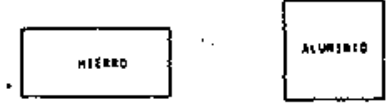

El cusdrudo es do aluminio y gesa 12 unidadets y, el otro, an de hivzro $y$ pesa 49 un id actes.

Ahors, se haca un agujero ciroulax en el trow da alyminzo $y$ un agujeto cuadrado en el de hierro de 1 ado iqual al disetro del agujero titcular. Si desplés de hacer los agujeros, el podazo de aluminio
restante pesa sólo? unidodes, Ceusnto pesars el trozo de hierzo remorido? ca.

Pot favor, indique elaramente camo llego a rapoust-

\section{APENDICE A}

Prueba: Medición del desarrollo intelectual (Parte B: control de variable)

La experiencia de hoy consiste en que Ud. estudie de que factores (o variables) depende el periodo de oscilación de un péndulo.

LLAMESE PERTODO DE OSCILACTOM DEL PENOULO AL TIEHPO QJJE OEMORA ESTE ER IA OESDE UNA POSICION EXTREMA A, AL OTRO EXTREMO A. Y LUEGo RETORNAR a a (VET PIg.).

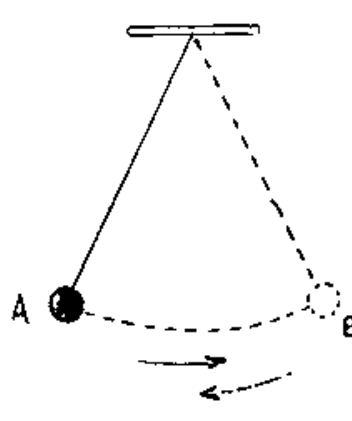

para ayudax an a trabajo 10 sugerimos que:

4) Haga primero yna liste do poablob factoras de los evolog Ud. inogina que depende ol pertodo del pénduio taenciona al meno. cuatrol.

b) Hago todas las experionciat necosariss pt za encontrar do que factores degendo el perlodo de un porndulo.

Deje constancis de codas las axperiencias realizadas, en su hoje de trabsjo.

NOTA, NO OCUPE KAS DE 40 NINUTOS EN LAS PAP TES A Y bi

a) Une rat deteruinados los anctores de los cuslat doponde el perfodo dal pjndulo, t-

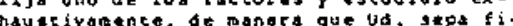
nalinente cówo depende la perlodo del pen. dulo dol tactor elegido.

Egta partin de eu trabajo deba anotarlas continuacion, con todo detalie. hasta llegar. su corelusión.

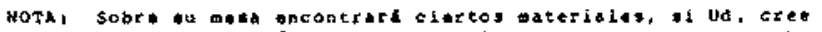
quo necasita of gateriales o inetrumentos para su trabajo. pued padirio a protetor.

\section{APENDICE B}

Modelo de Curso: Desarrollo niveles de razonamiento Versión 1980
HABIIIDADES INTELECTUALES
A DESARROLLAR (PROCESOS)

DISCAIXINAR ENTRE OBSERYAR $E$ INTERPRE TAR (PROCESO DE OBSERVAR)

RAZONAMIENTO PROPORCIOHAL (PROCZSO DE MEOTR)

RAZONAKIEATO PROPORC TONAL (PROCESO DE MEOIR) (PROCESO DE COMU( $(\mathrm{I}(\boldsymbol{\lambda} R)$.

RAZONAKIENTO PROPOS TC TONA RA ZONAMIEHTO PROPORCIOHAL (PROCESO DE MEDTR, COMUNICAR).

PAZONAMTENTO PROPOSICIONAL RAZONAKTENTO PROPORCIONAL.

PALTA DE INFORHACION EN UNA LINEA DE RAZONAKJERTO.

RAZOKAKI PNTO INOUCT ZVO, RAZOHAMIENTO DEOUCTIVO CADENA DR RAZONAMIENTO. (PROCESO DE INYERIR).

RAZONAMIEHTO OEDUCTIVO, CADENA DE RA ZONAKIENTO (PROCESO DE PREDECIR.

CONTROLANDO VARIABIES

RAZONAMIENTO OEDUCTIVO

\{PAOCESO CONTROL DE YARTABLES\}.

RAZONAMIENTO GIPOTETICO DEDUCTIVO RAZONAMIENTO PROPORCIONAZ.

SITUACION PROBLEUTICA

OBSERVANDO EL COMPORTAMIENTO DR OH PENDOLO ACO PLADO.

ESTUOIO SENCILLO OEL MOVIHIENTO OE UN CUERPO

MIOJEADO LAS DEFORMAC IONES SUPRIDAS POR UNA CQ LUMNA. OE AIRE.

MEDICION DE TIEMPOS CORTOS Y ROYTKIEMTO DE CAI OA Lxanz.

MIDIENDO CONO VARIA LA INTEMSIOAD LURIROSA COA LA DISTANCIA.

ESTUOSAMDO ZL. COMPORTAMEEXTO OE CJRCNITOS ELEC TRICOS.

INTIRIEHDO CONEXIONES OCULTAS RH UN CARCUITO eLzctrico.

PRED ELECTRICO, PREDICIEHDO EL COMPORTANIEHTO DE ON SISTEMA MECAKICO.

IOEHTIF CCAHDO Y COATROLANDO IAS YAGTABLES OB QUE DEPENDE EL TIEMPO DE GATADA EN UN PIAMO CEINADO. CORTHOZANDO IAS VAMIABLES DE QUE DEPEXOE ZT TERTODO DE UN PEMDULO.

MIDIENOO LOS ESPESORES DE UHA MOHOCAPA. 


\section{APENDICE C}

Prototipo de Actividades de Laboratorio Carso de Desarrollo Capacidad de Razonamiento

Prediciendo comportamiento de circuitos eléctricos

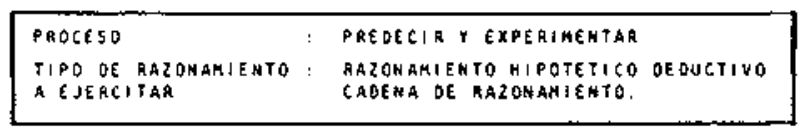

En la sesión pasada de taborarocio, vó esturo de dicado a tomptimentar con el comportamiento de los cireui tas eléctricos. En la sesión de Coloquio analizaron cuáles fueron las conclusiones mas importantes deducioas del com. portamienco de los circuitos y se condujo. la necesidad de formular un Nodelo Gualicativo para entender el comporta. mienco de nuevos circuitos.

El modelo cualitativo de circuito elfetrico desa. ceollado en la sesió ancerior se basó en los siquientes. cuatio oos culatios:

I. EXISTE UN*FLUJO EN UN CIRCUTTO CLEGTRICO CERRAOO

11. EL FLUJO SE CONSEAVA a TRAUES DE UH CIRCUITO

HII. LA MAGHITUO DEL FLUJO ESTA MEOIOA MEDIANYE EL BRILLO-

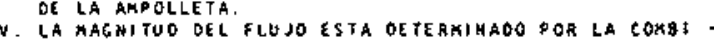
HACIOH DE COS ELEMEHTOS EN UN EIACUITO

Cl crabajo de esta semand consistirs en que lyo. los brillos de cona uno do las a varile. las en los eirevicos eléctricos dadose. "e para rea. izar dicha prediceión discone del modelo de clreuico, y de iada la información obtenida por Ud. ace tca del comporta. miento de circuiros.
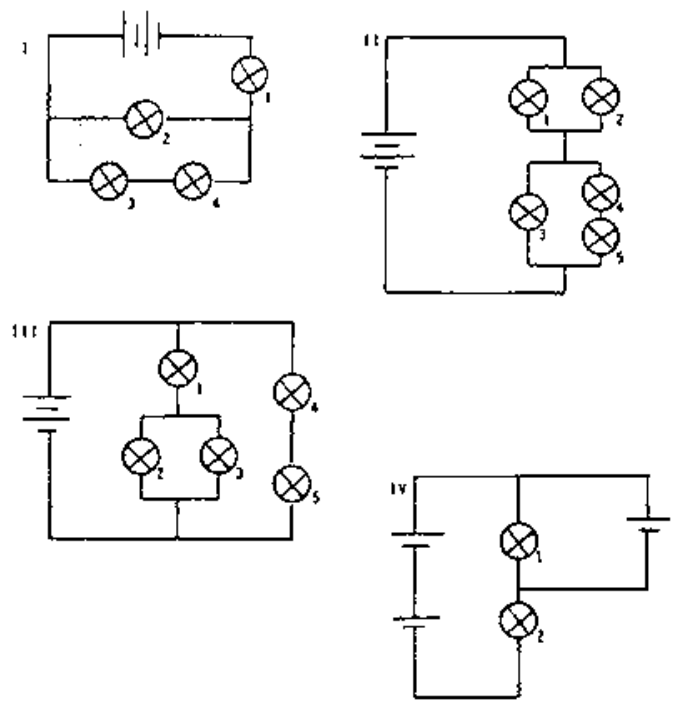

Una vel que que Ud. hoyd predicho el brillo de eada ampalleta. monce el circuito y compare lo observado conda ampollaca. para llevarlo o cabo recuerde ave las einco ampolletsas deben ser iguales.

En caso que no concuerden sus predicciones con los orillas obsefyodos, explique sl se debe que el mobelo fue mal tolicado o restmenze éste no es capaz de gredectir.

Al hacer su predictión y comparar con el verdadero*; comportamionco experimental. Ud. turo la oportunidad de poner prueb? su modelo de cirevita.

LSalib di roso su madelo?

\section{APENDICE D}

Protolipo de Actividades de Coloquio Curso de Desarrollo Capacidad de Razonamiento

\begin{tabular}{|c|c|c|}
\hline PROCESO & $:$ & PaE of CIR \\
\hline TIPO OE AAZOKANIEHTO & $:$ & RAZOKAHIENYO OEOUCTIVO \\
\hline A EJERCITAR & : & CADEKA DE RAZOWAHTEMTO \\
\hline
\end{tabular}

Lactividad propuesta se debe realizar en gru. pos de discusión de 4 alumnos cada uno. Como estrategia de

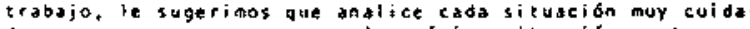
bosamente y que no arance a lo proxima situación, antes que discuca sw predicción con las grupos vecinos y por útitima con su profesor. CQUE LE SUCEOE AL BRHLLO OE LAS TOR ES CE RAADO?

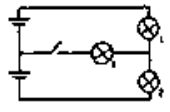

AABAS BATEAIAS Y AXPOLLETAS SON IGUALES, QQUE SUEEDE CON EL BRILLO OE LAS OOS AHPOLLETAS CUAKOO EL I $A T E$ GQUPTOR ES CEGRR. DO? LCUAL SERIA LA AESPUESTA A LA DREGUNYA AHTERIQR, SI LA PILA A, FUERA INGERTIOAT

LQUE LE SUCEDE AL BRILLO OE LAS AKPOLLETAS CUAKOO EL INTERRUP. TOR ES CEARADO?

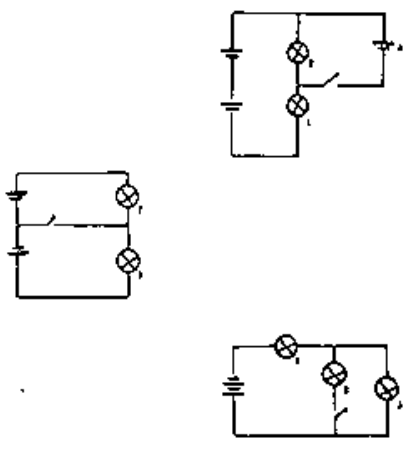

LQUE LE SUCEOE AL ORILLO DE LAS ANPOLLETAS CUANOO
TOR ES CERAADO?

OAOO EL SIGUIERTE GIRCHITO IN. OIQUE CONO SERA EL BRILLO OE LAS AAPOLLETAS 1 Y 2 C CUANOO EL $1 *$. TERRUPTO ESYA ESTA AOIERTO, Y. RRAOO.

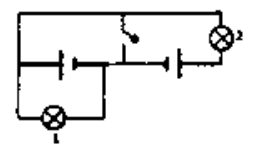

\section{APENDICE D}

Prototipos de Actividades de Laboratorio o Coloquio Curso Desarrollo Capacidad de Razonamiento

Interpretación de resultados experimentales

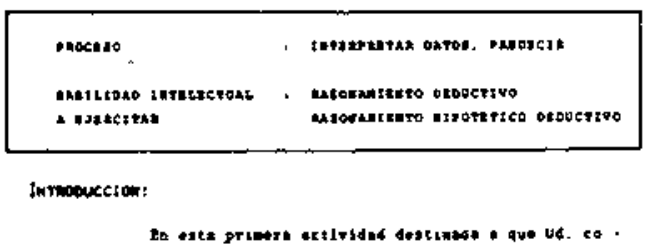

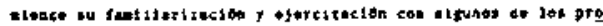

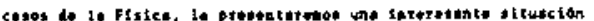

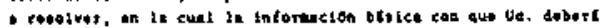
erebejer, ceti contenide on im grifleo.

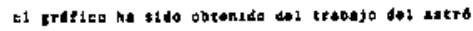

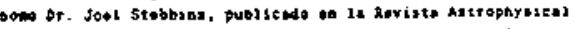

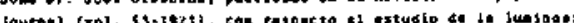

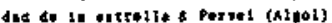

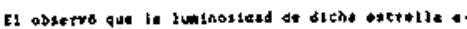

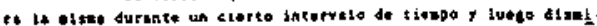

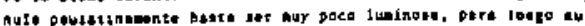

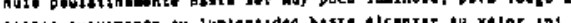

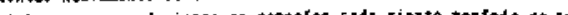

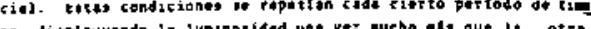


Lo que acabanos ae expresar esti representado en el grafaco luratrosidad-tiempo denouinado "curya de la luz de a Perse1 (Alq01)", que encontrata ás abajo.

Segutamente Ud. concordata con nosotros, que el comportaniento de la luminosidad de Algol, presenta un intere sante probleas. A continuacion encontrarta alguna de 123 posz bles explicaciones dadas por los cientsficos que parecen dar cuenta de esce hecho:

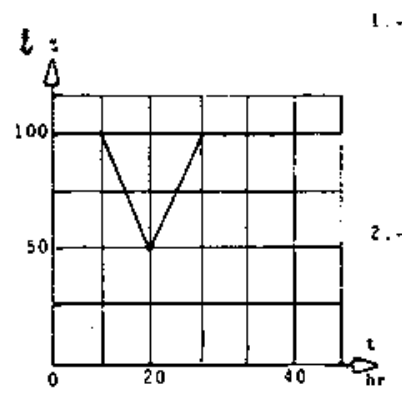

1. icubito es el valer de id luaing sigad gara

$\mathrm{t}=\mathrm{S} \mathrm{hr}$.

$\mathrm{c}=15 \mathrm{hr}$ :

$\mathrm{t}=20 \mathrm{hr}$

.............

. 40 ar

2.- QQue sucede can ia lutainosiozo entre

$\mathrm{t}=10 \mathrm{y} \mathrm{t}=20 \mathrm{mr} . \ldots \ldots$

...........................

$=20 \cdot y \mathrm{t}=30 \mathrm{hr} . \ldots \ldots \mathrm{C}$

3.- ipara que valor ae $t$ id lumino.

sidad alcanza su valor minino?

Ahord considere ios siguientes graficos t y 8 pard responder las preguntas 4 y 5 .
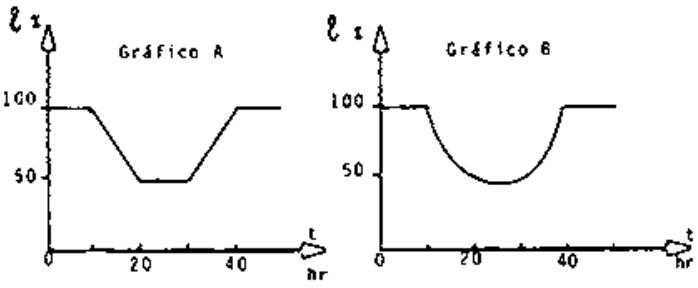

4) caracterice lo nejor que pueda la que sucese con la luminosida enzre $t=0$ y $t=40$ hrs. para casa grafico.

5) ¿En qué caso el ralor atinimo oe la luminosidad germanece cong tante durante un cierto interualo de ticago?

Le contidas de luz enfitida por ld estrelía grdeticamente disoinuye y luego vuelve a su valor nornal.

8. - Se trsta de dos estrelias que estan girsido en torno un gunto itjo y que cada clerto tiempo se ecligsan.

El trabsjo de esta sesion consistird entonces en abalizar sl estas posibles explfcaciones nos pernften saber algo ins sobre is estrelta. Pero antes reanos lgunas cosas prevías Que te ayudarbo l levar a cabo su trabajo.

Priaera que nada, he aqus dos definiclones sobre terminos que sparecerso ass adelante $y$ que es imperativo tener en cuente:

- Brillo: Llamaremos opillo a la cantiazd de iuz enttiok por unidad de drea.

- Lunfnosigad: Llagarenos luminosigad a la cancidad de lat que exile un objeto.

En sequndo iugar teataremos de fantiliarizario can

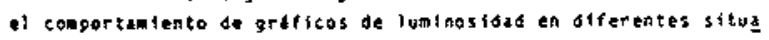
ciones para que Uo. desputs gueda interpretar sin dificultad el getfico se is estrella de kigot a ld iuz de ias postbles explica ciones bedas ancertormense.

En el grafico i se tha regresentado en el eje yertical 1. Iuminosidad oxde por und estrelly inaginaria fconsideranso que su vilor noximo corresponde al (005). Y en el eje horitontal. el tlengo expresado en hords.
Consiga dos moneras de igual tamaho. Hagalas gide posición. Yea ia ligura 1 .

haga girar las sonedas de no-

do que vo. ooserve un "eclido.

se cotal". es oectir que uns

moneda comience tager la a. tra hasta cubrirla cotalente.

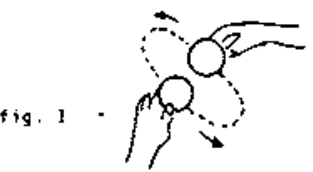

Si las gonedes entitieran luz, comos 6 el caso be las estrellas y construygramos un grafico luwinosifad-tieapo. nos encontrarianos aproximedemente con una curva de lum lnosidad como la presentadd continuacion.

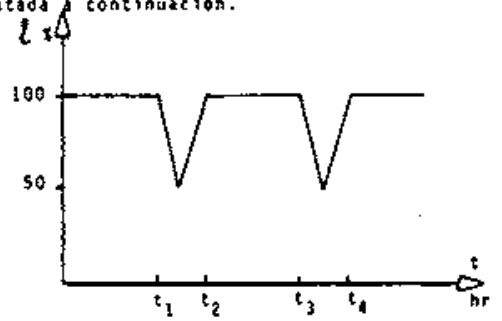

6) iEn cosi de los siguientes interralos llega igual cantioad de luz proventente de ambas monedas? Tarje lo que no corresponda.

$0 \times t_{1}: t_{1} \times t_{2}: \quad t_{2}+t_{3} ; \quad t_{3}=t_{4}$ tar en torno. un punto fijo y ublquese frente a ellas sin canbiar 
7) iEn qué interratos las nonedas se eclipsan garcialmente o totalemente?

a) ¿kasta qué valor baja ia luainosidad durante los ecliposes? ipot qué?

9) (9or que el graftco gara esta situación no poorfa haber sioo como:

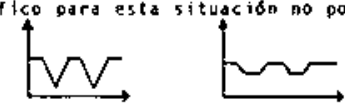

Si existiera solo teclipse parcialu. es decir. que para el adservador las monedas no blegan a cubrirse tolalmente, como por ejemglo se ilustra en la figura 2.

201 Ciudl de las curvas de luz oibujadas a continuacion represen caris mejor aste caso? (ayidase reproductendo esta situacion con las monedas?

$\left.C^{f i g}\right)^{2}$
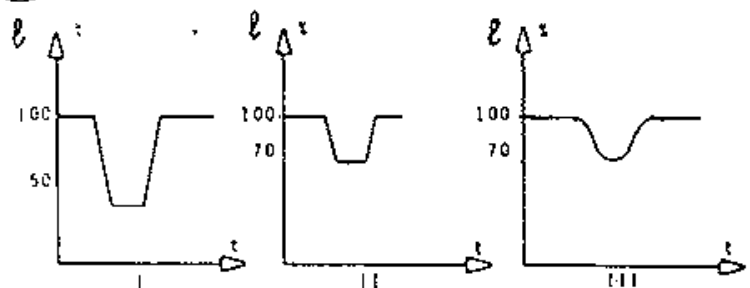

Combie una de las monedas por oted mas pequeha $y$ quéralss de agdo que Ud. abserve un "eclipse tocal" (suponga que atrods tienen igual briliol.

11.- itudl de los grfficos dados anteriormente. I. II, Ili, representarla este caso? de arguantos que justifiquen su eleceión.

Cuando ta moneda mas gequera pasa adelante de la mis grande, como por ejemplo en la forma liustrada en le figura 3 . se observa ia que se detromina un "eclipse andlar".

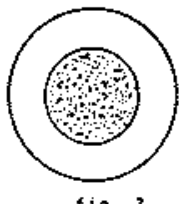

Reprosuzed un eclipse anular con sus mionedas. En tal caso:

12) LCusi oe los graficos 1, 11, III. reprasentarfa esta nueva situacion? de argumentos que Justifiquen su eleccion.

En el grafico Que bamos a continuación se ha tibu jado uns curya de luz correspondiente d dos estrellas (imaglospida) oue se eclipsan peribalcamente garz un observasor fljo en la tie.

Q ' $\mathrm{C}^{\mathrm{a}}$;

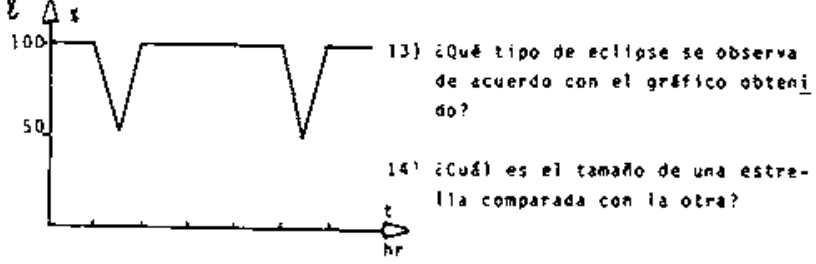

Si se ooseryd aue id luminosidad disataye en ca Ga eclipse hasta un sor.

151 ique puede deducir acerca de i. lueinosidod de una estreila conparada con ia otra?

16) si supanemos que und estrellid es ats lustnosa que ia otra gero siempte iguai tanatio, ique modfflcactones harta al gra fico \$\$rO?

17) A continuacion se indican cuatro curvas de iuginosidad rs. tiempo IY, $Y, Y I, Y I t$.

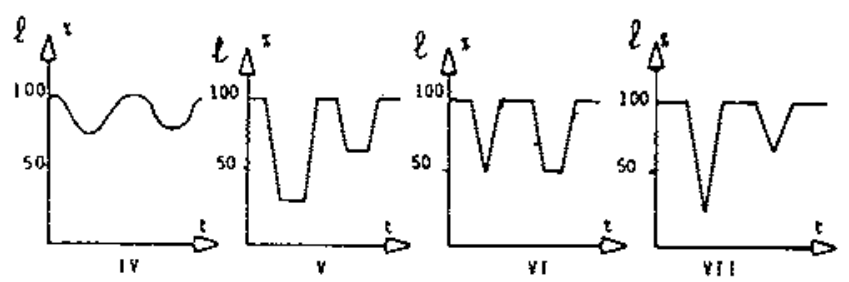

A cada una de las siguientes situaclones aescritas $(A, B, E)$, he gales corresponder uno de los grsficos anteriores, IV, Ұ. YI, ҰII. Justifique cada electión.

A. - En un caso se observa eclipse tozal y luego eclipse anular. Las estrellas son de oistinto tamaho $y$ distinta luminosidad

E. En amoos casos se observa eclipse total. las estrelias son de iqual tamaño, peto una más luainosa que la otra.

c.- Se obserya que los ecilpses son parciales, las estrollas son de lgual idegatio y de lgual luninosload

18) Andice thord ia curva de luminosidad correspondiente a th taso reel. cono io es la curva $\beta$ perset (algol).

Determine les caracterfsticas generales a partir de id information entregate por el grafico y las suposictones he chas por lob. oel sisteme oincrio que constituye la estrella de Algol.

19) Leodrid deductr algunt caracterfstica de Aigol, tentendo pre sente lo grtmerd suposicion dada li comiento 